\title{
Inward propagating chemical waves in Taylor vortices
}

\author{
Barnaby W. Thompson, ${ }^{1}$ Jan Novak, ${ }^{2}$ Mark C. T. Wilson, ${ }^{3}$ Melanie M. Britton, ${ }^{2}$ and Annette F. Taylor ${ }^{1, *}$ \\ ${ }^{1}$ School of Chemistry, University of Leeds, Leeds LS2 9JT, United Kingdom \\ ${ }^{2}$ School of Chemistry, University of Birmingham, Birmingham B15 2TT, United Kingdom \\ ${ }^{3}$ School of Mechanical Engineering, University of Leeds, Leeds LS2 9JT, United Kingdom
}

(Received 22 December 2009; published 28 April 2010)

\begin{abstract}
Advection-reaction-diffusion (ARD) waves in the Belousov-Zhabotinsky reaction in steady Taylor-Couette vortices have been visualized using magnetic-resonance imaging and simulated using an adapted Oregonator model. We show how propagating wave behavior depends on the ratio of advective, chemical and diffusive time scales. In simulations, inward propagating spiral flamelets are observed at high Damköhler number (Da). At low $\mathrm{Da}$, the reaction distributes itself over several vortices and then propagates inwards as contracting ring pulses-also observed experimentally.
\end{abstract}

DOI: 10.1103/PhysRevE.81.047101

PACS number(s): 82.40.Ck, 47.20.Qr, 47.54.- r, 47.70.Fw

Spatiotemporal patterns in reaction-diffusion systems have been extensively studied [1,2], with an increasing interest in coupling fluid flow with such systems. The interactions between stirring effects of advection-producing large scale homogenization - and the sharp chemical gradients produced by reaction-diffusion processes generate complex patterns in both chemical and biological systems $[3,4]$. For the prototypical Belousov-Zhabotinsky (BZ) reaction, these advection-reaction-diffusion (ARD) structures have been studied in packed bed reactors [5] and, more recently, in a magnetohydrodynamically generated chain of counterrotating vortices [6,7]. Here, vortex flow induces significant front wrinkling, and front structures and propagation velocities are further affected by chaotic mixing due to lateral vortex oscillations and axial flows, producing complex patterns. Such vortices in other autocatalytic systems produce similar effects $[8,9]$ with an enhancement of front velocity by the flow field, limited by diffusion across vortex separatrices. This agrees with previous analytical and numerical studies for autocatalytic "burn" combustion fronts [10-12].

Common to these systems are different qualitative regimes of pattern formation, dependent on dimensionless numbers based on the vortex scale: a Péclet number $(\mathrm{Pe})$ relating advective to diffusive time scales, a Damköhler number (Da), relating chemical to advective time scales, and the ratio of vortex size $(L)$ to front width $(\lambda), \xi=L / \lambda$. For large vortices, $\xi>1$, when advection dominates $(\mathrm{Pe} \gg 1, \mathrm{Da}$ $\ll 1$ ), the reaction zone is distributed across several cells simultaneously. In contrast, when reaction and diffusion play a larger role $(\mathrm{Da} \gg 1)$ a more defined "flamelet" propagates across a cell (see [9]).

ARD structures in laminar cellular flows provide useful analogs to model various complex systems, for example, dynamics of turbulent flows and quenching in combustion [11]. To date, no general theories of these systems exist, and further experimental, numerical and analytical studies are required. In this Brief Report, we examine cellular ARD structures within a well-established experimental system: the Couette cell in the regime of steady counter-rotating toroidal

\footnotetext{
*Corresponding author; a.f.taylor@leeds.ac.uk
}

Taylor vortices [13] (Fig. 1). A Couette cell has been exploited previously to examine reaction-diffusion waves in open conditions [14] and to examine front propagation enhanced by mixing, but in a turbulent regime [15]; here we employ magnetic-resonance imaging (MRI) to visualize both the steady vortex flow field [16] and the structure and velocity of chemical waves $[17,18]$ propagating through itreaction-diffusion pulses rather than one-off fronts. Laboratory studies are supported by a numerical model based on the empirically derived Oregonator [19] incorporating a vortex flow. This reproduces key behavior and further explores the effect of flow strength on chemical structures and velocity.

For experimental MRI studies, a Couette cell with a gap of $3.0 \mathrm{~mm}$ was filled with well-mixed oscillatory BZ reagents. The manganese-catalyzed system was used due to its suitability for MRI study [17]. The inner cylinder was rotated above a critical velocity to establish steady Taylor vortices (Fig. 1). All MRI measurements were performed on a Bruker DMX300 spectrometer, operating at a proton resonance frequency of $300 \mathrm{MHz}$ at $289.4 \pm 0.2 \mathrm{~K}$. Chemical waves were visualized using a spin-echo imaging sequence [Fig. 2(a)]. Typical flow field velocities in three dimensions were visualized using a pulsed gradient spin-echo (PGSE) velocity imaging sequence [20] [Fig. 1(c)].

A typical time series of images in Fig. 2(a) shows a pulse propagating through a series of Taylor vortices, resulting in inward propagating rings distributed across several cells simultaneously. Effective front velocities increase with increasing $\omega$ (and hence increasing vortex velocity) and are significantly enhanced compared to the reaction-diffusion velocity of $0.137 \mathrm{~mm} \mathrm{~s}^{-1}$, in general agreement with $[6,8,9]$.

For simulations the two-variable Oregonator model [19] is used, with the Tyson-Fife scalings [21] amended to scale space by the gap between the cylinders. Time is scaled by the chemical time scale, $k_{\mathrm{c}} B$, rather than advective time scales often employed [22]. This gives the following ARD equations:

$$
\frac{\partial u}{\partial t}=\frac{1}{\mathrm{PeDa}} \nabla^{2} u-\frac{1}{\mathrm{Da}} \underline{\phi} \cdot \underline{\nabla} u+\frac{1}{\varepsilon}\left\{u(1-u)-f v \frac{(u-q)}{(u+q)}\right\},
$$




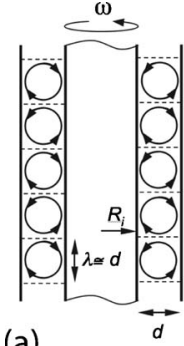

(a)

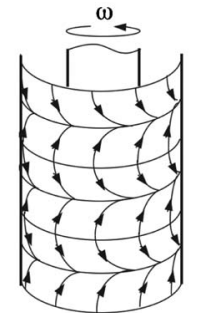

(b)

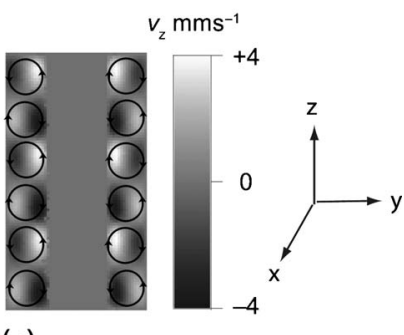

(c)

FIG. 1. Taylor vortices within a Couette cell. (a) Schematic of meridional plane cross section. Angular speed $\omega$, gap $d$, wavelength $\lambda$ and inner radius $R_{\mathrm{i}}$. (b) Three-dimensional (3D) toroidal structure. (c) MRI velocity map for meridional plane cross section, showing axial $(z)$ velocity $\left(\mathrm{mm} \mathrm{s}^{-1}\right)$. The field of view shown is $20 \mathrm{~mm}$ (vertical) $\times 11 \mathrm{~mm}$ (horizontal) with a pixel size of 195 $\times 203 \mu \mathrm{m}$. PGSE gradient pulses were applied axially with a maximum strength of $0.25 \mathrm{~T} \mathrm{~m}^{-1}, 1 \mathrm{~ms}$ duration, $50 \mathrm{~ms}$ separation and eight velocity encoding steps. $\omega=2.6 \mathrm{~Hz}, d=3.0 \mathrm{~mm} \approx \lambda$, and $R_{\mathrm{i}}$ $=2.5 \mathrm{~mm}$.

$$
\frac{\partial v}{\partial t}=\frac{1}{\mathrm{PeDa}} \nabla^{2} v-\frac{1}{\mathrm{Da}} \underline{\phi} \cdot \underline{\nabla} v+u-v
$$

These explicitly provide $\mathrm{Da}=k_{\mathrm{c}} B L / \Phi_{0}$, and $\mathrm{Pe}=\Phi_{0} L / \mathrm{D} . u$ and $v$ are the autocatalyst $\mathrm{HBrO}_{2}$ and the oxidized metal catalyst $\mathrm{Mn}^{3+}$, respectively. $\Phi_{0}$ is a characteristic flow speed in the meridional (axial-radial) plane, $D$ is a typical diffusion coefficient, and $L$ is the annulus. $f, \varepsilon$ and $q$ are chemical parameters incorporating key rate constants and concentra-

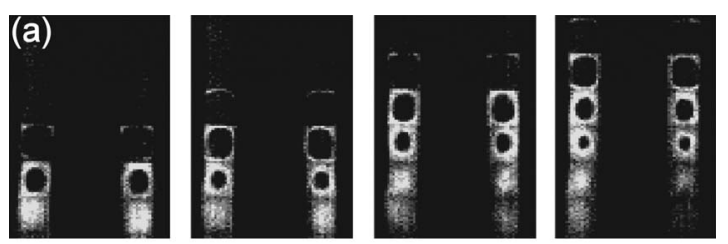

(b)

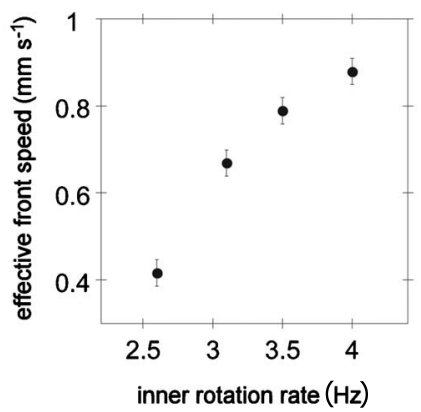

FIG. 2. Experimental results. (a) MRI image in meridional $(y-z)$ plane. The field of view shown is $20 \mathrm{~mm}$ (vertical) $\times 13 \mathrm{~mm}$ (horizontal) with a pixel size of $176 \times 203 \mu \mathrm{m}$. Image contrast is produced by differences in $T_{2}$ magnetic-resonance relaxation times for water protons surrounding $\mathrm{Mn}^{2+}$ (dark) $/ \mathrm{Mn}^{3+}$ (light) ions. A recovery time of $100 \mathrm{~ms}$ was used, giving an imaging time of 6.4 s. $\quad \omega=2.6 \mathrm{~Hz}$. $\left[\mathrm{Mn}^{2+}\right]=6 \times 10^{-4} \mathrm{M}, \quad\left[\mathrm{CH}_{2}(\mathrm{COOH})_{2}\right]$ $=0.15 \mathrm{M}, \quad\left[\mathrm{BrO}_{3}^{-}\right]=0.05 \mathrm{M}, \quad\left[\mathrm{Br}^{-}\right]=0.06 \mathrm{M}, \quad\left[\mathrm{H}_{2} \mathrm{SO}_{4}\right]=0.6 \mathrm{M}$, $\left[\mathrm{H}_{3} \mathrm{PO}_{4}\right]=2.8 \mathrm{M}$. (b) Plot of effective front speed vs inner cylinder rotation rate, $\omega$.

(a)

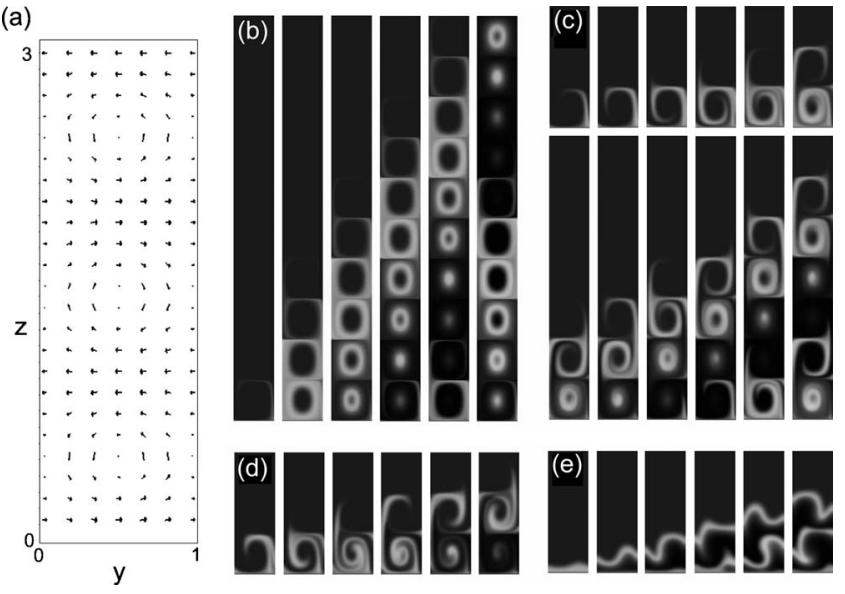

FIG. 3. Simulated cell. (a) Plot of 2D flow vector $\phi$ for first three vortices. Arrows indicate strength and direction of flow field. (b)-(e) time snapshots (from left to right) of front propagation up the tube for different values of $\mathrm{Da} / \mathrm{Pe}$. Light areas indicate high $\left[\mathrm{Mn}^{3+}\right]$. (b) $\mathrm{Da}=0.3(\mathrm{Pe}=1500) ; \tau=1,4,6,8,10$, and 15. (c) $\mathrm{Da}$ $=1.5(\mathrm{Pe}=300) ; \tau=2,2.5,3,3.5,4,4.5$ (top row), 5, 6, 7, 8.5, 10, and 14 (bottom row). (d) $\mathrm{Da}=3.0(\mathrm{Pe}=150) ; \tau=3.5,4.5,5.5,6.5$, 7.5, and 8.5. (e) $\mathrm{Da}=30(\mathrm{Pe}=15) ; \tau=3,6,9,12,15$, and 18 .

tions. $k_{\mathrm{c}} B$ is effectively the rate of production of the inhibitor, bromide ion. Experimentally, typically $k_{\mathrm{c}} B \cong 0.1 \mathrm{~s}^{-1}$, i.e., a typical chemical time scale, $\tau_{\mathrm{r}} \cong 10 \mathrm{~s}$. The cylinder's rotation rate is sufficiently fast for azimuthal variations in concentration to be neglected, and therefore a twodimensional (2D) dimensionless vector field $\phi=\left(\Phi_{z}, \Phi_{y}\right)$ is used to represent the flow in the meridional plane only. Rather than solve the Navier-Stokes equations for $\phi$, however, we construct the simplest function that produces a cellular flow structure close to that observed in the TaylorCouette system, taking care to satisfy the no-slip condition on the cylinder surfaces, located at $y=0$ and $y=1$,

$$
\phi_{z}=\sin [(2 y-1) \pi] \sin [\pi z / \alpha],
$$

$$
\phi_{y}=\frac{1}{2 \alpha}(1+\cos [(2 y-1) \pi]) \cos [\pi z / \alpha] .
$$

Figure 3(a) illustrates this geometrical model of the timeindependent streamlines for steady flow. The parameter $\alpha$ is the eddy aspect ratio, determined experimentally to be unity. A grid convergence study determined 100 grid points across the gap to be sufficient, with 1000 points used axially to capture a series of ten vortices. Reaction-diffusion waves were initiated by a bottom boundary layer of high $\mathrm{HBrO}_{2}$. For a particular set of chemical parameters equivalent to the oscillatory regime $(f=1, \varepsilon=0.25, q=0.0008)$, we examine behavior as we vary the eddy strength, $\Phi_{0}$ and hence Da and Pe, with $\mathrm{Da} \sim 1 / \mathrm{Pe}$ [see Figs. 3(b)-3(e)]. (Note that, as a comparison, azimuthal Damköhler and Péclet numbers based on the rotating cylinder's surface speed are approximately $3 \times 10^{-4}$ and $3 \times 10^{5}$, respectively).

In the $\mathrm{Da} \ll 1, \mathrm{Pe} \gg 1$ regime $(0.03<\mathrm{Da}<0.5,900<\mathrm{Pe}$ $<15$ 000), advection within cells dominates [Fig. 3(b)]. Autocatalyst entering the cell perimeter is advected rapidly 
along vortex outer streamlines and then diffuses across the separatrix, distributing the reaction across a number of cells very rapidly. Diffusive and reactive time scales are longer, and so propagation toward the cell center, across streamlines, is slower. This results in a circular wave front propagating inwards via a reaction-diffusion mechanism, followed by the associated wave back as the clock resets. This produces a contracting ring of oxidized catalyst. After a refractory period, the next wave front enters the outer part of the cell, setting the process off again, while the previous ring is still contracting. By this time, a large number of cells ahead have been contaminated by the original front. This results in reverse target patterns within each coupled cell, driven by advection but limited by diffusion across cells with closed streamlines. This corresponds to the experimental image in Fig. 2(a); here $\Phi_{0}=4.0 \mathrm{~mm} \mathrm{~s}^{-1}$ giving (for $k_{\mathrm{c}} B=0.1 \mathrm{~s}^{-1}, L$ $=3.0 \mathrm{~mm}$, and $\left.D=2 \times 10^{-3} \mathrm{~m}^{2} \mathrm{~s}^{-1}\right) \quad \mathrm{Pe}=6000$ and $\mathrm{Da}$ $=0.075$, a good agreement.

For Da $>1$, [Fig. 3(c)], however, inward propagating spirals of two sharply separated phases are observed. Reaction and (to a lesser extent) diffusion in all directions compete more equally with slower advection round streamlines. Autocatalyst propagates inwards on similar time scales as it rotates around streamlines, hence spiraling into the cell center. This flamelet winds in and disappears in approximately equal time as the contracting rings above. This is expected as inward motion is limited by reaction diffusion; notably, in this time period significantly fewer cells ahead have been contaminated due to much slower advection to the boundary with the next cell; i.e., the total "front" structure length over multiple cells is determined by the consumption time of already contaminated cells. A more complex "hollow" spiral structure is observed if $\mathrm{Da}$ is increased further [Fig. 3(d)]; the spiral rotates more slowly and thus there is time for a "split" as the reaction-diffusion wave propagates in opposing (primarily radial) directions.

As Pe approaches zero, Da $\gg 1$ [Fig. 3(e)] the vortex strength becomes comparatively very weak and the system approaches the geometrical optics regime of sharp planar reaction-diffusion fronts [10]. Figure 4 shows effective pulse velocity down the cell axis, $c_{f}$, for different values of Péclet numbers. With increasing flow rate, i.e., increasing $\mathrm{Pe}$ (decreasing Da), $c_{f}$ increases monotonically with a power law of 0.39 , due to the limiting effect of diffusion across the separatrices. As we are fitting over a large range of $\mathrm{Da}$, our exponent is an intermediate value of those previously predicted by an effective diffusion approach driven by the rate of contamination of additional cells [10-12], where $c_{f} \sim \Phi_{0}^{3 / 4}$ at low Da and $c_{f} \sim \Phi_{0}^{1 / 4}$ at high Da.

In summary, we have demonstrated that MRI is uniquely able to visualize both the chemistry and flow in these systems, enabling a clear picture of the dynamics. We observe a distributed reaction zone with inward propagating rings. Simulations using our adapted Oregonator and geometrical flow function capture key experimental behavior and allow us to explore a wide range of steady vortex regimes. Notably, this model is more sophisticated than previous "burn" models as it simulates pulses. In agreement with the literature

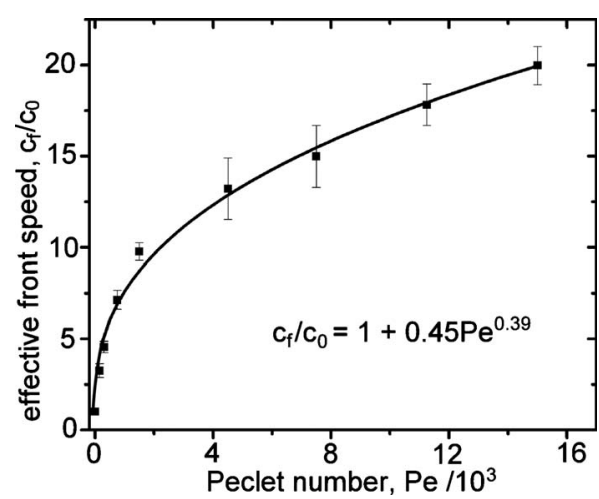

FIG. 4. Dependence of effective front velocity (scaled by reaction-diffusion velocity $\mathrm{c}_{0}$ when $\mathrm{Pe}=0$ ) on Péclet number. (There is no significant difference in velocities calculated from points down the center axis or on the outer edge of the meridional plane. This is also true for velocities of first compared to subsequent pulses).

[6-12], for our large vortex regime, with increasing flow rate and hence decreasing Da (and increasing Pe), we observe the transition from laminar reaction-diffusion waves to spiral flamelets and on to distributed reaction inward ring structures, with an increase in effective front velocity. For the current experimental setup, we were unable to explore the full set of numerically observed behavior by altering the flow rate as the minimum critical flow rate for steady cells was 2.5 $\mathrm{Hz}$ and MRI artifacts were observed above $4.0 \mathrm{~Hz}$. However, these other regimes might be observed by varying the chemistry (and hence Da independently of Pe) or altering the MRI imaging sequence. We also note that, importantly, Pocheau and Harambat [9] found a sensitivity of front trajectory to boundary layers in cellular flow in a Hele-Shaw cell. They thus proposed local 3D kinematic modeling of least time trajectories to fully account for cell geometry. We recognize that the assumption of fast azimuthal flow in our model becomes questionable close to the surface of the stationary cylinder; while good agreement between the model and experiments suggests this is not significant for the cases presented here, the sensitivity to this feature should be explored. Using MRI, effective diffusion coefficients can be obtained. The influence of boundary layers will thus be the subject of future work.

Steady vortices generated in this Taylor-Couette reactor system provide an analogous cellular ARD regime to those generated magnetohydrodynamically [6-9]. This suggests the underlying ARD mechanisms in cellular flow are robust, and can be applied to a variety of flow environments. The combination of MRI in a Couette cell-with its large number of different flow regimes [13]_ and numerical modeling of the corresponding flow field provide a unique platform for the quantification of the influence of flow on autocatalytic chemical reactions.

\section{ACKNOWLEDGMENTS}

The authors thank the EPSRC (Grants No. EP/F048777/1 and EP/F050410/1) and the Royal Society for funding. 
[1] Chemical Waves and Patterns, edited by R. Kapral and K. Showalter (Kluwer, Dordrecht, 1995).

[2] I. R. Epstein, J. A. Pojman, and O. Steinbock, Chaos 16, 037101 (2006).

[3] P. Andresen, M. Bache, E. Mosekilde, G. Dewel, and P. Borckmanns, Phys. Rev. E 60, 297 (1999).

[4] I. R. Epstein, Nature (London) 374, 321 (1995).

[5] M. Kaern and M. Menzinger, Phys. Rev. E 60, R3471 (1999).

[6] M. S. Paoletti and T. H. Solomon, Phys. Rev. E 72, 046204 (2005).

[7] M. E. Schwartz and T. H. Solomon, Phys. Rev. Lett. 100, 028302 (2008).

[8] A. Pocheau and F. Harambat, Phys. Rev. E 73, 065304(R) (2006).

[9] A. Pocheau and F. Harambat, Phys. Rev. E 77, 036304 (2008).

[10] M. Abel et al., Chaos 12, 481 (2002).

[11] N. Vladimirova et al., Combust. Theory Modell. 7, 487
(2003).

[12] M. Abel et al., Phys. Rev. E 64, 046307 (2001).

[13] C. D. Andereck, S. S. Liu, and H. L. Swinney, J. Fluid Mech. 164, 155 (1986).

[14] Q. Ouyang et al., Phys. Lett. A 134, 282 (1989).

[15] P. D. Ronney, B. D. Haslam, and N. O. Rhys, Phys. Rev. Lett. 74, 3804 (1995).

[16] J. D. Seymour, B. Manz, and P. T. Callaghan, Phys. Fluids 11, 1104 (1999).

[17] M. M. Britton et al., J. Phys. Chem. A 109, 8306 (2005).

[18] A. Tzalmona et al., Chem. Phys. Lett. 174, 199 (1990).

[19] R. J. Field and R. M. Noyes, J. Am. Chem. Soc. 96, 2001 (1974).

[20] P. T. Callaghan, Principles of Nuclear Magnetic Resonance Microscopy (OUP, Oxford, 1991).

[21] J. J. Tyson and P. C. Fife, J. Chem. Phys. 73, 2224 (1980).

[22] Z. Neufeld, P. H. Haynes, and T. Tel, Chaos 12, 426 (2002). 